\title{
Attentional guidance of the eyes by contextual information and abrupt onsets
}

\author{
MATTHEW S. PETERSON and ARTHUR F. KRAMER \\ Beckman Institute, University of Illinois at Urbana-Champaign, Urbana, Illinois
}

\begin{abstract}
Contextual cuing is a memory-based phenomenon in which previously encountered global pattern information in a display can automatically guide attention to the location of a target (Chun \& Jiang, 1998), leading to rapid and accurate responses. What is not clear is how contextual cuing works. By monitoring eye movements, we investigated the roles that recognition and guidance play in contextual cuing. Recognition does not appear to occur on every trial and sometimes does not have its effects until later in the search process. When recognition does occur, attention is guided straight to the target rather than in the general direction. In Experiment 2, we investigated the interaction between memorydriven search (contextual cuing) and stimulus-driven attentional capture by abrupt onsets. Contextual cuing was able to override capture by abrupt onsets. In contrast, onsets had almost no effect on the degree of contextual cuing. These data are discussed in terms of the role of top-down and bottom-up factors in the guidance of attention in visual search.
\end{abstract}

Recently, Chun and Jiang (1998; see also Chun \& Jiang, 1999) have demonstrated a phenomenon known as contextual cuing in which the global patterns of scenes are able to implicitely ${ }^{1}$ guide attention. Contextual cuing is a memory-based phenomenon in which the global patterns must be learned before guidance can occur. In a typical experiment, half of the displays are unique to each trial, whereas the remaining displays are repeated throughout the experiment. Several different repeated displays are used. For the repeated displays, the locations, but not the identities, of the target and distractors are held constant in each example. For instance, if the task is to find a left or right tilted $\mathrm{T}$ among rotated Ls, the identity of the target (left or right tilted T) will vary in each trial of the repeated configuration, but the location of the target $\mathrm{T}$ will remain constant.

The contextual cuing effect develops rapidly with minimal practice, and responses to the repeated configurations are quicker than responses to the new displays by the second block of trials, which is the first instance in which the displays are repeated. For the most part, subjects are often unaware that any repetitions have occurred. When an explicit recognition test of the displays is performed after the experimental search trials, subjects who report being consciously aware of the repetitions during the experiment show identical contextual cuing benefits to those that were unaware of the repetitions. Chun and Jiang (1998) suggest that contextual cuing is an example of instance-based

This research was supported by Grant AG14966 from the National Institute on Aging and a cooperative research agreement with the Army Research Laboratory (DAAL01-96-2-0003). The authors thank Shawn Bolin for his assistance in running subjects. Correspondence should be addressed to M. S. Peterson or A. F. Kramer, Beckman Institute, University of Illinois, 405 North Mathews Ave., Urbana, IL 61801 (e-mail: mspeters@uiuc.edu or akramer@s.psych.uiuc.edu). learning (Logan, 1988), in which encountering a previously observed situation automatically activates past solutions and actions. In the example of contextual cuing, the presentation of a repeated configuration automatically activates past instances of attentional guidance. That is, the recognition process that guides attention is automatic and does not require conscious effort. Results similar to contextual cuing have been found by Logan (1998) using a different task. In Logan's task, subjects were asked to determine whether a word was from a target category. Subjects' responded more quickly when the word occurred in the same spatial location in the testing phase as it had during the training phase.

Other forms of attentional guidance can occur in the absence of learning. For example, the occurrence of new objects in a display, even when such objects are irrelevant to the task, can capture attention (Yantis \& Jonides, 1984, 1990) as well as the eyes (Irwin, Colcombe, Kramer, \& Hahn, 2000; Kramer, Hahn, Irwin, \& Theeuwes, 1999, 2000; Theeuwes, Kramer, Hahn, \& Irwin, 1998; Theeuwes, Kramer, Hahn, Irwin, \& Zelinksy, 1999). Other aspects of the environment, such as uniquely colored or moving objects, can also grab attention, especially when subjects have an attentional set for these aspects of the environment (Folk \& Remington, 1998, 1999; Folk, Remington, \& Johnston, 1992; Yantis \& Egeth, 1999). Attentional capture in these instances appears to be due to discontinuities or the appearance of new objects in the display, whereas contextual cuing can occur in instances in which there are no unique local or global patterns that are common from trial to trial. ${ }^{2}$

One question that remains unanswered is how contextual cuing guides attention. There would appear to be at least two important components of contextual cuing: recognition and guidance. Recognition entails the identification of the current context of the scene and matching this information 
with previously stored instances of the familiar context. Guidance entails the use of stored knowledge concerning scene layout to accurately guide attention to locations in the scene that are likely to be important. Contextual cuing does not appear to immediately guide attention to the target, or at least not on every occasion. Chun and Jiang (1998, Experiment 4) investigated how contextual cuing affected search slopes during the course of an experiment. Although search slopes decreased as subjects gained more experience with the repeated displays, the search slopes never reached zero and were still quite steep (approximately $27 \mathrm{msec} / \mathrm{item}$ ) at the end of the experiment. This would appear to rule out the possibility that contextual cuing always rapidly guides attention directly to a target. However, other possibilities exist in the role that recognition and guidance play in the contextual cuing effect. When recognition occurs, it might be completed before search has begun, or recognition might be completed later during the search process. Although Chun and Jiang (1998, Experiment 5) showed that contextual cuing can develop rapidly enough to occur even for displays flashed for $200 \mathrm{msec}$, this does not preclude the possibility that sometimes contextual recognition develops more slowly and has its effects later in the search process. While finding contextual benefits for briefly flashed displays would suggest that contextual cuing can develop rapidly and that guidance is highly accurate, it could also be the case that guidance is accurate on only a small proportion of trials. In this case, guidance might be imperfect and guide attention in the direction or general vicinity of the target, allowing peripheral vision to identify the target.

Contextual cuing enables search to be more efficient, and the shallower search slopes Chun and Jiang (1998) found for the repeated displays would suggest that fewer items on average were examined to find the target. Unfortunately, since the slopes reflect the average behavior over the course of the experiment or a block of trials, it is impossible to determine through response times the sources of the steepness of the slopes. That is, several quite different explanations can account for the pattern of response times observed during contextual cuing. These possibilities include (1) a mixture distribution, in which the response times are from trials in which recognition guided attention immediately to the target and trials in which recognition failed, (2) a spatially imprecise guidance mechanism that directed attention to the general vicinity of the target, allowing peripheral vision to guide attention to the target and reduce the number of items searched, and (3) a guidance mechanism that was highly accurate and a recognition mechanism that sometimes did not recognize the context until many of the items had already been searched.

Given that there is considerable evidence that attention precedes an eye movement (Deubel \& Schneider, 1996; Hoffman \& Subramaniam, 1995), using eye movements to study contextual cuing allows us to determine on each trial where attention has been (fixation location), the inaccuracy of attentional guidance (the angular error of a saccade and distance of a fixation from the target), and the num- ber of items that were attended before the target was found (the number of fixations).

Each of these possible explanations for the steepness of the search slopes predicts a unique pattern of response times and eye movements. The simplest hypothesis to test is whether the pattern of response times is due to a mixture of trials in which recognition fails on a substantial proportion of trials. In this case, the eyes should fail to go immediately to the target on a number of trials, and the first saccade on these trials should show no bias in the direction of the target. That is, trials in which the eyes fail to go immediately to the target should be indistinguishable from trials in which a new configuration was presented. Conversely, if the attentional guidance mechanism is spatially imprecise, then a bias should be apparent in the direction of the first saccade and the distance of the first fixation when the eyes fail to initially land on the target. Another possibility is that contextual cuing does not always develop until later during the search process. If it is possible for recognition to occur (or have its effects) later during the search process, then repeated configurations should show a benefit in the manual response times and the number of fixations even when the eyes did not go immediately to the target.

In the following experiments, we addressed how recognition and guidance produce the contextual cuing effect by monitoring subjects' eye movements during the search process. In Experiment 1, we examined how contextual cuing affects the trajectory and accuracy of the initial saccade relative to the location of the target and the number of saccades that must be made until the target is found. In Experiment 2, we compared the memory-based guidance of contextual cuing and the stimulus-driven guidance of abrupt onsets (i.e., new objects) and examined how they interact to influence scanning behavior during the search process.

The design of Experiments 1 and 2 was adapted from Chun and Jiang (1998) with several important modifications. To accomplish our goal of collecting meaningful scan patterns, we needed to change the displays such that they would encourage subjects to search serially. To achieve this end, the stimuli were smaller than those used by Chun and Jiang (1998) and were designed to encourage foveation. In addition, we used a target and distractor set (rotated Ts and Ls) that has a history of producing serial searches (Wolfe, Cave, \& Franzel, 1989). In Experiment 2, onsets were created through the use of a premask display. Premasks marked the locations where all but one of the items in the display would occur. Therefore, when the search display was presented, the item without the premask occurred as a sudden onset. To make Experiments 1 and 2 comparable, we also used the premask displays but did not include an onset in Experiment 1.

\section{EXPERIMENT 1}

Chun and Jiang (1998, Experiment 4) ruled out the possibility that contextual cuing guides attention immediately to the target such that it is the first and only item exam- 
ined. Although they found that repeated configurationshad a shallower search slope than the new configurations, the search slopes for the repeated configurations were never flat, as would be expected if the target was the first and only item examined. In Experiment 1, we investigated the nature of the recognition and guidance processes that lead to contextual cuing. If the recognition process can occur or be completed after search has begun, then the contextual cuing effect should still be evident even in trials in which the first saccade failed to land on the target. That is, relative to responses to the new configurations, responses to find the target should be quicker and fewer saccades should be made, even when trials in which the target is immediately found are ruled out.

Another question concerns the accuracy of the attentional guidance mechanism. Contextual cuing might guide attention directly to the target, or it might be less accurate and guide attention in the direction or to the vicinity of the target. If the attentional guidance occurs but is spatially imprecise, then the angular distance of the first saccade relative to the target and the distance of the first fixation from the target should be smaller for the repeated configurations then for the new configurations.

\section{Method}

Subjects. Eighteen students (6 males and 12 females) from the University of Illinois were paid to participate in the experiment. The average age of the subjects was 21.3 years.

Apparatus. A Gateway Pentium 133-MHz computer with a 19in. SVGA color monitor running custom software was used to present the stimuli, control the timing of the experimental events, and record the subjects' response times. Eye movements were recorded with an Eyelink tracker (SR Research Ltd.) with $250-\mathrm{Hz}$ temporal resolution and a $0.2^{\circ}$ spatial resolution. The system used an infrared video-based tracking technology to compute the center and size of the pupils in both eyes. An infrared head motion tracking system tracked head motion. Even though head motion was measured, in the present study the head was stabilized by means of a chinrest. The chinrest was located $53.3 \mathrm{~cm}$ from the monitor.

Design. Two variables of interest were combined in a $16 \times 2$ orthogonal design: block and configuration (new or repeated). For purposes of analysis, the 16 blocks were collapsed into four epochs, with each epoch consisting of data from 4 blocks.

Stimuli. The stimuli consisted of white Ts and Ls approximately $0.44^{\circ}$ tall and $0.44^{\circ}$ wide, drawn on a black background. There were 11 distractors and 1 target always present in the display. Targets consisted of $90^{\circ}$ and $270^{\circ}$ rotated Ts with their stems pointing to the right or to the left. Distractors were normal or mirror-imaged Ls rotated $0^{\circ}, 90^{\circ}, 180^{\circ}$, or $270^{\circ}$, and premasks were formed from the line segments of every possible target and distractor. An example of a premask display is shown in the top panel of Figure 1, and an example of a stimulus display is shown in the bottom panel.

Displays were generated by randomly placing items on an imaginary $12 \times 12$ grid, with the constraint that no items could occur in the central four locations near the fixation cross. The width and the height of the grid were $32^{\circ}$, and the minimum distance between any two stimuli was $2.7^{\circ}$. Twelve repeated configurations were randomly generated for each subject, and a single example of each configuration occurred in each block.

Procedure. Each trial began with the presentation of a fixation cross. The subjects pressed the space bar to begin the trial while fixating the cross, and the trial continued only if the subject was fixating within $2.5^{\circ}$. After the space bar had been pressed, the premask display was presented for $1,000 \mathrm{msec}$ and was then replaced by the search display. Stimuli in the search display were formed by removing line segments from the premasks, and, at the same time, portions of the central fixation cross were removed and signaled to the subjects that they could begin their search. If the subjects moved their eyes from the fixation cross before it had changed (i.e., during the premask display), a tone sounded and the trial was aborted. The subjects pressed the "z" or "/" keys to identify whether the target present was a left- or right-rotated T. Response keys were counterbalanced across subjects.

At the end of the experiment, the subjects performed a recognition task. The recognition task consisted of 12 new and 12 repeated displays of premask placeholders, and the subjects were asked "How certain are you that you've seen this display before?" and were asked to rate each display on a 5 -point scale, with $1=100 \%$ certain and $5=$ never seen the display.

The subjects served in a single 1-h session consisting of 16 blocks of 24 trials, for a total of 384 trials.

\section{Results}

All repeated-measures analyses of variance (ANOVAs) and contrasts were tested using Greenhouse-Geisser corrected $p$ values, and unless otherwise stated, were 2 (new or repeated configuration) $\times 4$ (epoch) repeated-measures designs. For each subject, manual responses times greater than twice the mean for each cell in each block were considered outliers and were excluded from analysis.

Manual responses. Manual response times are shown in Figure 2. As noted previously by Chun and Jiang (1998, 1999), response times to repeated configurations were quicker than those to new configurations $[F(1,17)=$ $127.70, p<.01]$, and the main effect of epoch shows that manual responses became quicker as the subjects gained experience with the task $[F(3,51)=15.81, p<.01]$. Configuration and epoch failed to interact. However, a finer grained analysis comparing the first 4 blocks (the first epoch) revealed an interaction between block and configuration $[F(3,51)=3.00, p<.05]$. Like Chun and Jiang (1999), we found that contextual cuing can develop rapidly within the first few repetitions.

Eye movements. A fixation was counted as landing on a target if it was within $1.3^{\circ}$ of the target. The median number of fixations before the eyes landed on the target can be seen in Figure 3. Fewer fixations were required for the repeated configurations then for the new configurations $(M$ $=5.1$ and 6.3 , respectively) $[F(1,17)=121.19, p<.01]$, suggesting that fewer items needed to be examined in the repeated displays before the target was found. The number of fixations also decreased as a function of epoch $[F(3,53)=4.21, p<.01]$. Configuration failed to interact with epoch; however, like the response time data, a finer grained analysis of the first epoch revealed an interaction between block and configuration $[F(3,53)=3.68, p<$ $.05]$. The median number of fixations in each condition for each subject was positively correlated with the mean response times $(R=.90)$ and accounted for $81 \%$ of their variance. On average, each fixation increased manual responses by $215 \mathrm{msec}$.

Both the distance of the first fixation $[F(1,17)=7.8, p<$ $.05]$ and the angle of the first fixation from the target 


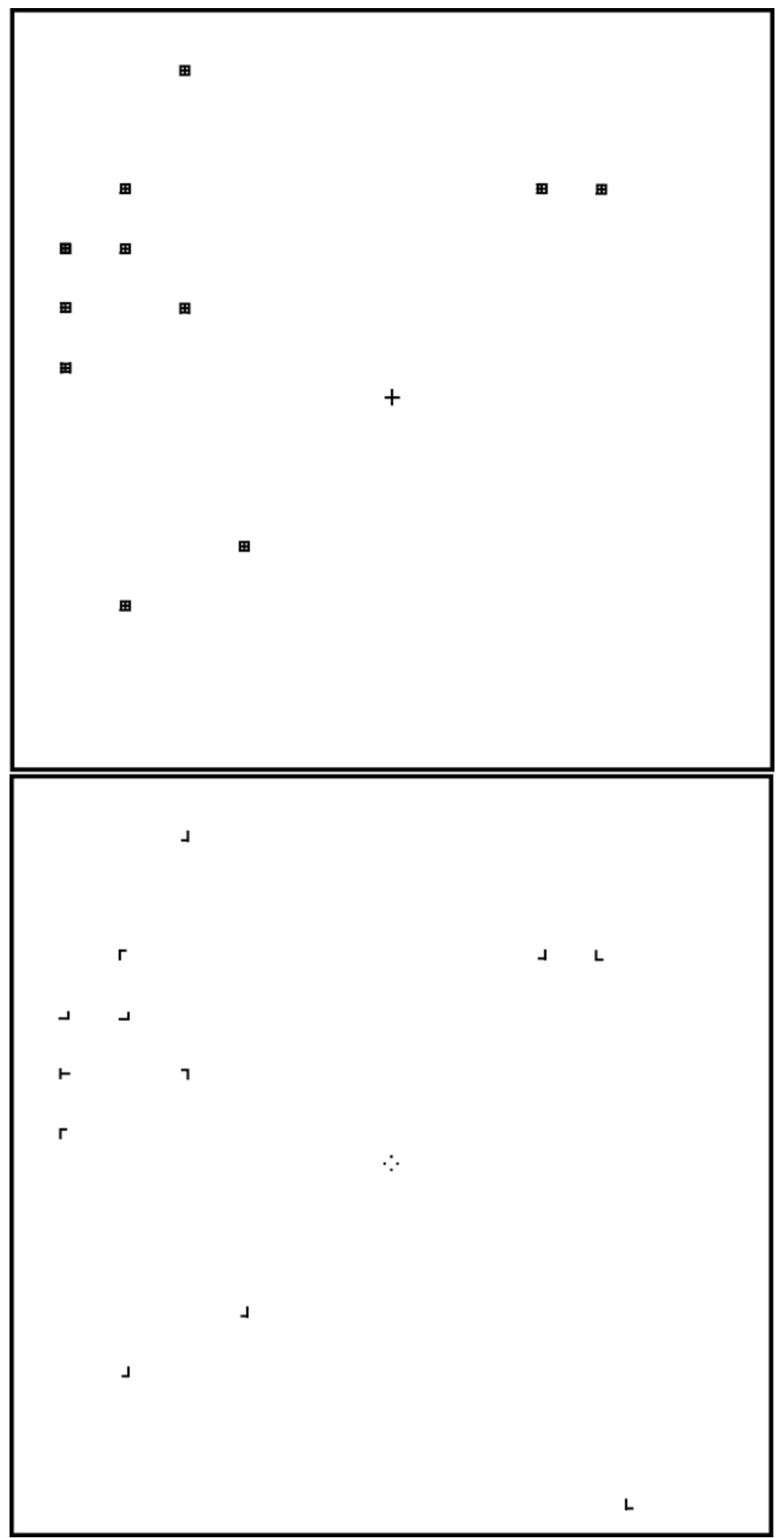

Figure 1. The top panel shows an example of a premask display similar to those used in Experiments 1 and 2. The stimulus example shown in the bottom panel is from an onset present trial from Experiment 2 . The onset stimulus is the upright $L$ in the lower right corner of the bottom panel. 


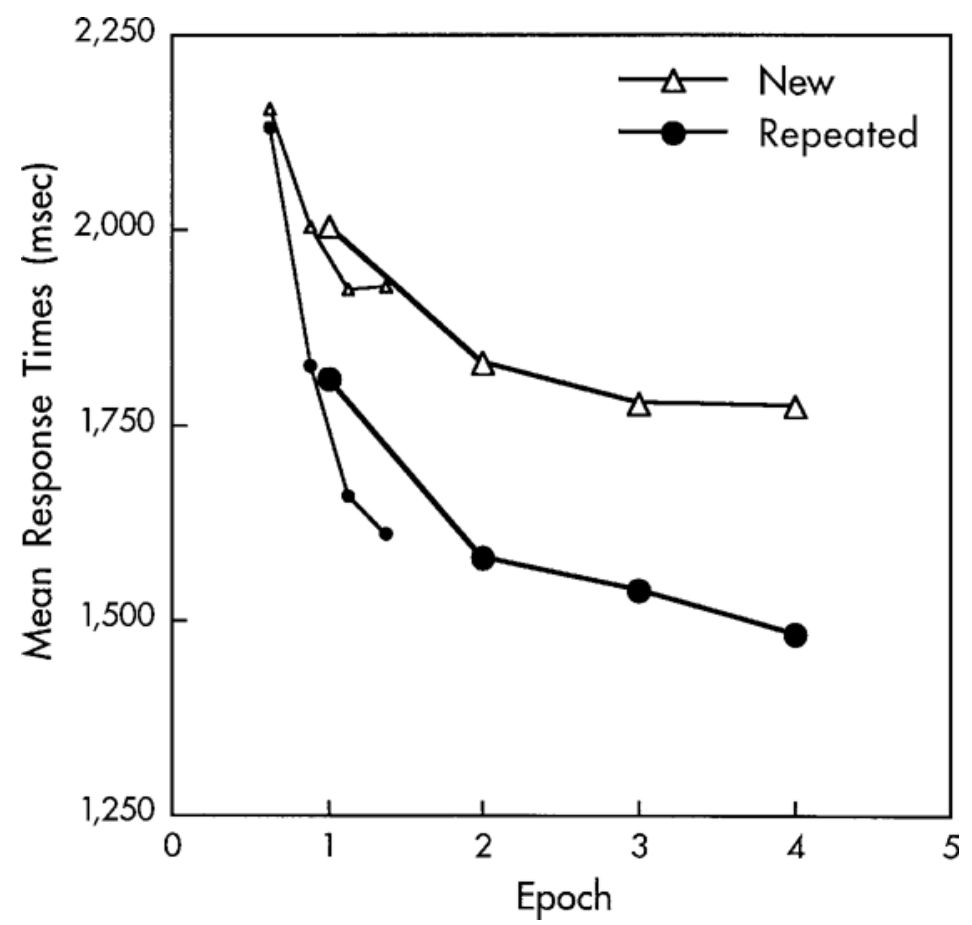

Figure 2. Mean response times across epochs for the repeated and new configurations of Experiment 1. The thin lines on the left of the graph are from the four blocks of trials that make up the first epoch.

$[F(1,17)=5.63, p<.05]$ were smaller for the repeated configurations $\left(M \mathrm{~s}=13.3^{\circ}\right.$ visual angle and $81^{\circ}$ radially $)$ than for the new configurations $\left(M \mathrm{~s}=14.3^{\circ}\right.$ visual angle and $84^{\circ}$ radially). At first glance, this might appear to suggest that the attentional guidance mechanism is not always accurate and at times might guide attention to the general vicinity of the target rather than directly to the target. That is, although the first saccade on average landed closer to the target for the repeated configurations, the average distance was still quite large. However, as can be seen in Figure 4, the proportions of initial fixations that landed on the target were greater for the repeated configurations than for the new configurations $(M \mathrm{~s}=11.3 \%$ and $7.1 \%$, respectively) $[F(1,17)=23.1, p<.01]$, and, when trials in which the eyes went directly to the target were removed from the analyses, the distance and angle of the first saccade were no longer significant $\left(M \mathrm{~s}=14.9^{\circ}\right.$ visual angle and $86^{\circ}$ radially for repeated configurations, and $M \mathrm{~s}=15.1^{\circ}$ visual angle and $87^{\circ}$ radially for the new configurations). That is, the trials in which the eyes landed on the target (within $1.3^{\circ}$ ) first accounted for the fixation distance and angle effects. This suggests that the attentional guidance mechanism is accurate in terms of spatial location, and, when recognition occurs, attention is guided directly to the target.

The number of fixations before the eyes landed on the target was reanalyzed after removing trials in which the eyes went directly to the target on the first saccade. If recognition can take place or be completed after search has commenced, then the median number of fixations should be smaller for the repeated configurations than for the new configurations, even when the trials in which the eyes first landed on the target are taken into account. Even with the trials in which the eyes went initially to the target were removed from the analysis, fewer fixations were required to find the target in the repeated then in the new

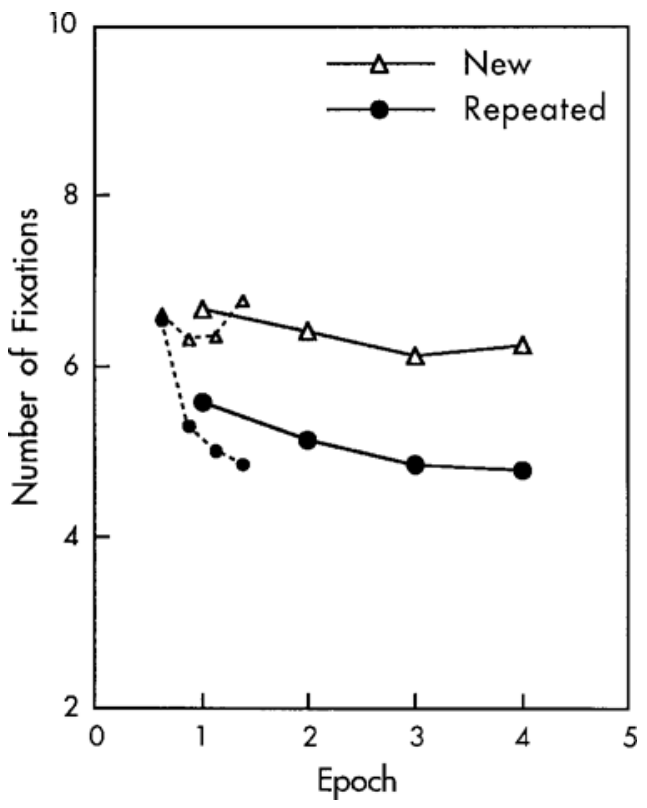

Figure 3. Number of fixations needed to find the target in Experiment 1 graphed by the type of configuration and epoch. 


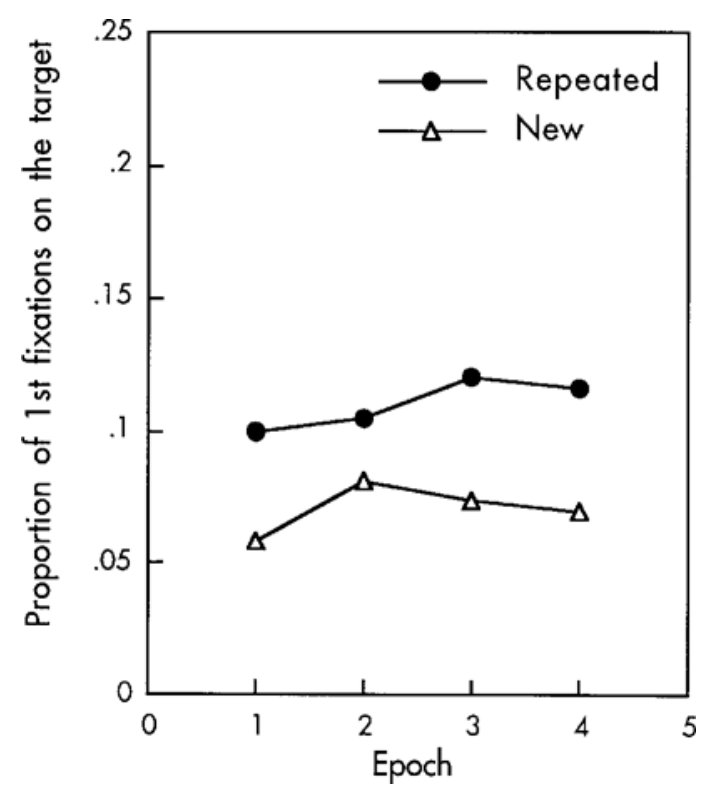

Figure 4. The proportions of initial fixations that landed on the target for the new and repeated configurations of Experiment 1.

configurations $(M \mathrm{~s}=5.3$ and 6.5 , respectively) $[F(1,17)=$ $64.5, p<.01]$.

To look at the effect of experience on the number of saccades required to find the target, the number of fixations were analyzed in a three-way repeated-measures ANOVA with the type of configuration (new or old), number of fixations needed to find the target on each trial (1-14 fixations), and block (16 blocks) as factors. The proportions of fixations for the new and repeated configurations are shown in Figure 5. As expected, the type of configuration present $[F(1,17)=19.3, p<.01]$ affected the proportion of fixations, and the proportion of fixations were not evenly distributed $[F(13,221)=55.60, p<.01]$.
Of more importance is the fact that the type of configuration and the number of fixations interacted $[F(13,221)=$ $10.0, p<.01]$, and planned comparisons showed that the target was more likely to be found within the first three fixations when a repeated configuration was presented than when a new configuration was presented [one-tailed $t(221)=1.80, p<.05]$.

Recognition test. The subjects were more likely to rate the repeated configurations as familiar than the new configurations $(M \mathrm{~s}=2.79$ and 3.47 , respectively, with $1=$ $100 \%$ certain and $5=$ never $)[t=4.58, p<.01]$. The degree of recognition was calculated by subtracting the mean rating score for the repeated configurations from the mean rating score of the new configurations for each subject. No significant correlation was found between the contextual cuing benefits (repeated - new)/response times) or the proportional cuing benefits [(repeated - new)/repeated response times] and the degree of recognition. This suggests that contextual cuing is an automatic process that does not require explicit recognition.

\section{Discussion}

The results of Experiment 1 show that the recognition process in contextual cuing is able to occur or be completed after search has already begun. Fewer fixations were needed to find the target when the subjects viewed the repeated configurations even when their eyes failed to immediately go to the target. When recognition takes place, the guidance mechanism is able to precisely locate the target: No bias toward the target was found when the initial saccade landed elsewhere. Taken as a whole, contextual cuing is able to guide attention to the important areas of a scene-any inaccuracies in the guidance of attention are due to a failure in recognition rather than inaccuracies in attentional deployment.

Contextual cuing is not the only phenomenon in which stimulus properties can automatically guide attention. Abrupt onsets, or visual transients, can automatically guide

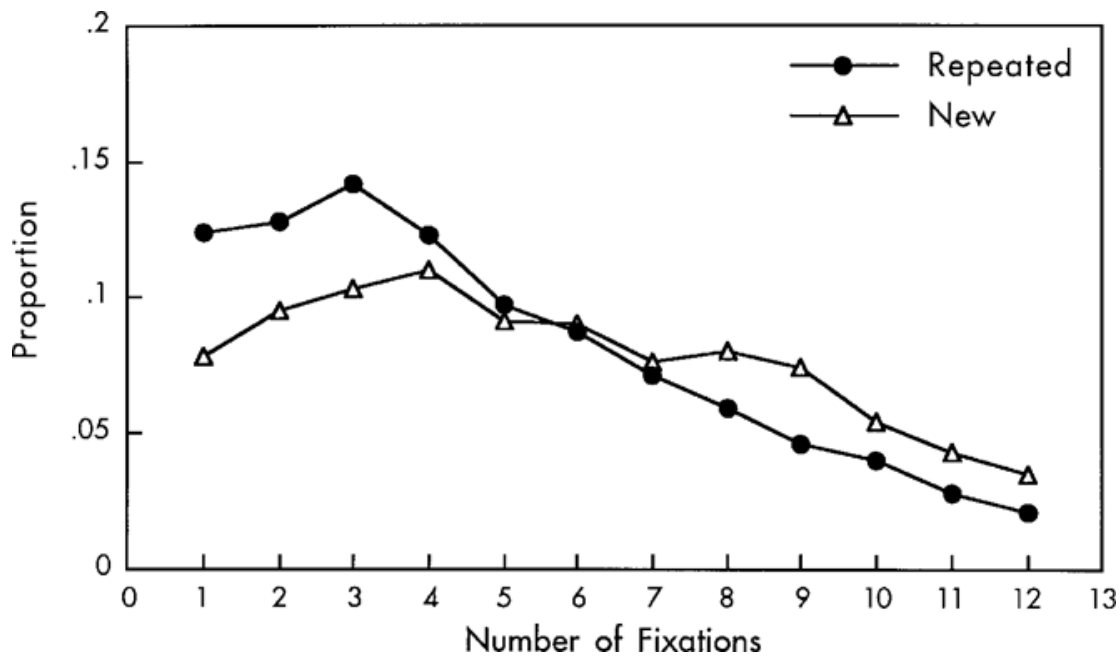

Figure 5. The probability density functions for the number of fixations needed to find the target for the new and repeated configurations of Experiment 1. 
attention to potentially important locations in a scene (Yantis \& Jonides, 1984, 1990; but see Folk et al., 1992) and can even cause the eyes to move to unintended locations. Theeuwes et al. (1998) have shown that task-irrelevant abrupt onsets can capture attention and the eyes even when the target is the only colored item in the display. In Experiment 2 , we examine how the memory-based guidance of contextual cuing and the stimulus-driven guidance of abrupt onsets interact and compete to guide attention.

\section{EXPERIMENT 2}

Although both contextual cuing and abrupt onsets can guide attention during visual search, it is not clear how they are related or how they might interact. One possibility is that contextual cuing might be able to override capture by abrupt onsets, leading response times to show a smaller onset cost and causing fewer initial saccades to be made to the onset for repeated displays than for new displays. Indeed, other manipulations, such as shifting attention to the location of a potential target prior to the appearance of an onset distractor (Theeuwes et al., 1998; Yantis \& Jonides, 1990) and increasing the amount of change in a display (Martin-Emerson \& Kramer, 1997; Miller, 1989), have been shown to reduce capture by abrupt onsets. Another possibility is that abrupt onsets might interfere with contextualcuing, such that when onsets capture attention, no evidence for contextual cuing is observed after the eyes go to the onset. This might take place if the occurrence of the abrupt onset interfered with the association of the display and the memory representations of the display configuration, leading to a failure of recognition-based atten-

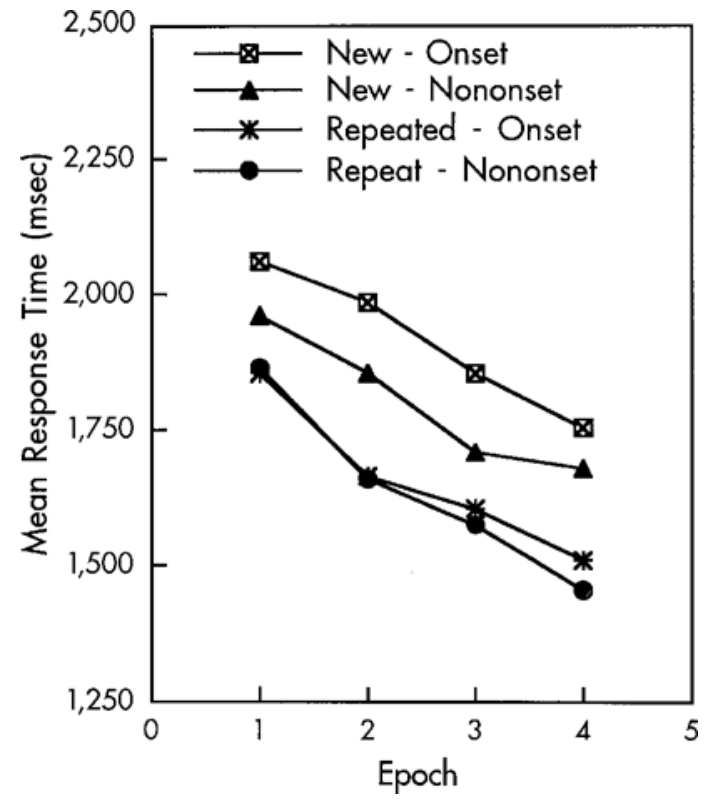

Figure 6. Mean response times across epochs for new and repeated displays when onsets were present and absent in Experiment 2. tional guidance. A third possibility is that onsets might interfere with learning of the repeated configurations. This last possibility would lead to a smaller, or even an absent, contextual cuing effect, even on trials in which the onset is not present. Such a possibility would be consistent with previous reports of reduced evidence of learning in the absence of attention (Logan, Taylor, \& Etherton, 1999).

Experiment 2 was identical to Experiment 1, except that half of the trials contained an abrupt onset. Each block of trials contained a nononset and an onset example of each repeated display.

\section{Method}

Subjects. Twenty-one students ( 7 males and 14 females) from the University of Illinois were paid to participate. The average age of the subjects was 21.3 years.

Design. Three variables of interest were combined in a $8 \times 2 \times 2$ orthogonal design: block $\times$ configuration (new or repeated) $\times$ onset presence (present or absent). For analysis purposes, the eight blocks were collapsed into four epochs, with each epoch consisting of data from two blocks

Stimuli. The stimuli were identical to those used in Experiment 1, except that the onset trials contained one less premask than the nononset trials. The onset was the item in the display that had not been preceded by a premask placeholder. The object that would serve as the onset was randomly chosen for each trial, with the constraint that the onset could never be the target.

Procedure. The procedure was identical to that used in Experiment 1, except that each block consisted of twice as many trials (48) as had occurred in Experiment 1. Each block contained an onset and a nononset trial for each repeated configuration, and the order of trial presentation was randomly determined, with the constraint that the onset and nononset examples of a configuration never occurred consecutively.

The subjects served in a single 1-h session consisting of eight blocks of 48 trials, for a total of 384 trials.

\section{Results}

Manual responses. Manual response times are shown in Figure 6. As in Experiment 1, repeated configurations were responded to more quickly than were new configurations $(M \mathrm{~s}=1,651$ and $1,859 \mathrm{msec}$, respectively) $[F(1,20)=116.86, p<.01]$, and response times decreased in later epochs $[F(3,60)=33.38, p<.01]$. As can be seen in Figure 6, response times were slower when onsets were present $(M \mathrm{~s}=1722$ and $1788 \mathrm{msec})[F(1,20)=$ $11.91, p<.01]$. More importantly, onset presence and configuration interacted $[F(1,20)=4.66, p<.05]$, and contrasts revealed that onsets affected response times only when the subjects viewed new configurations $[F(1,20)=$ $13.9, p<.01$, for new configurations; $F<1$, for repeated configurations], suggesting that contextual cuing is able to override capture of attention by abrupt onsets. Like Experiment 1 , the interaction between epoch and configuration failed to reach significance. However, unlike Experiment 1 , a finer grained analysis of the first few blocks failed to find an interaction between block and configuration $[F(1,20)=1.27, p>.10, \beta=.821]$, possibly because the first instances of some of the repeated configurations contained onsets. Another possibility is that because the onset and nononset versions of a configuration were 


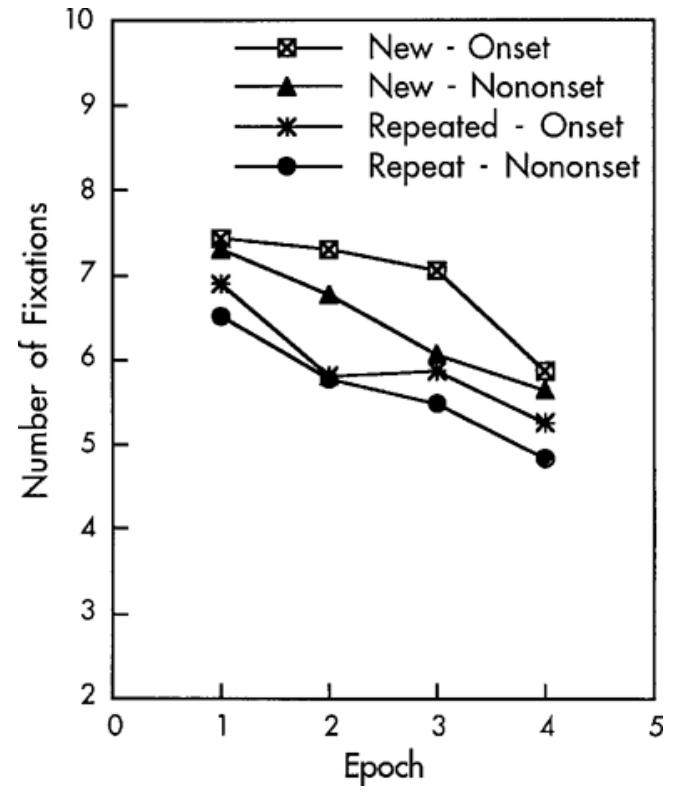

Figure 7. The number of fixations needed to find the target across epochs for new and repeated displays when onsets were present and absent in Experiment 2.

placed randomly within a block, the first instance of a nononset (or onset) version of a repeated configuration could be either the first time or the second time that that configuration was encountered within a block of trials. Combined with rapid contextual learning (see Experiment 1 ), this made detecting a block $\times$ configuration interaction less likely than in Experiment 1.

Eye movements. The median number of fixations until the eye landed on the target can be seen in Figure 7. As in the Experiment 1, fewer fixations were required to find the target in the repeated displays than in the new displays $(M \mathrm{~s}=5.8$ and 6.8 , respectively) $[F(1,20)=77.80 p<$ $.01]$, and fewer fixations were required to find the target as the number of epochs increased $[F(3,60)=9.93, p<$ $.01]$. Like the mean response times, onset presence increased the number of fixations $(M \mathrm{~s}=6.17$ and 6.44 , for repeated and new configurations, respectively) $[F(1,20)=$ $6.10, p<.05]$. Unlike the response time data, configuration and onset presence failed to interact.

The median distance of the first fixation from the target and from the onset were analyzed to determine whether onset presence affected the first saccade. To determine whether onset presence affected the distance and angle of the first fixation from the onset, a boot-strapping technique was used for the nononset trials in which a random distractor was picked as the nononset equivalent of the onset. Unlike Experiment 1, no significant effects were found for the distance of the first fixation from the target. However, the eyes landed closer to the onset when the subjects viewed new configurations than when they viewed repeated configuration $\left(M \mathrm{~s}=13.5^{\circ}\right.$ and $14.3^{\circ}$, respectively) $[F(1,20)=10.16, p<.01]$, suggesting that contextual cuing was able to partially override capture by onsets.
The proportion of first fixations that landed on the target tells a different story. Unlike the distance effects, the type of configuration affected the first saccade, with the first fixation more likely to land on the target when the subjects viewed a repeated configuration than when they viewed a new configuration $(M \mathrm{~s}=.095$ and .064 , respectively) $[F(1,20)=16.16, p<.01]$. As can be seen in Figure 8 , the first fixation was more likely to land on the target at later epochs $[F(3,60)=3.74, p<.05]$.

The evidence that contextual cuing can override onsets continues to be seen in the proportion of initial fixations that landed on the onset. In the analysis, we used the same boot-strapping technique as that used for the distance data. The eyes were less likely to land initially on the onset or its nononset equivalent when the subjects viewed repeated configurations than when they viewed new configurations $(M \mathrm{~s}=.058$ and .071 , respectively $)[F(1,20)=$ $4.75, p<.05]$. A planned comparison of the effects of the type of configuration on onset present trials also showed a decrease in the number of initial saccades that went to the onset when the subjects viewed repeated configurations relative to when they viewed new configurations $(M \mathrm{~s}=.077$ and .094 , respectively) $[F(1,20)=5.28, p<$ $.05]$. In addition, the eyes were more likely to land on an onset when it was present than on the nononset equivalent $(M \mathrm{~s}=.085$ and .046 , respectively) $[F(1,20)=10.51, p<$ $.01]$.

One question that arises is, When an onset captures attention, does it disrupt contextual cuing altogether, or is contextual cuing able to recover after the first misfixation to the onset? To test this, we analyzed only the trials in which the eyes went directly to the onset. Since capture by onsets was a relatively rare event $(5.8 \%$ for the repeated configurations), the data were collapsed across the differ-

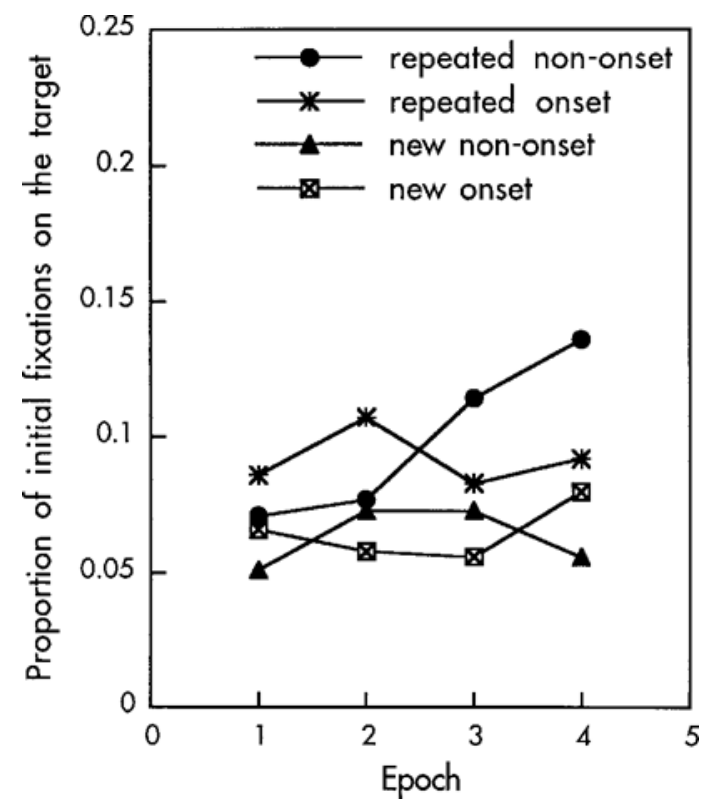

Figure 8. The proportions of initial fixations that landed on the target in Experiment 2. 
ent blocks of trials. Both the distance of the second fixation from the target and the proportion of second fixations that landed on the target following a fixation to the onset were analyzed. The type of configuration had no effect on the distance of the second fixation from the target. However, even when the eyes went initially to the onset, fewer additional fixations were needed to find the target in the repeated configurations than in the new configuration $(M \mathrm{~s}=5.9$ and 6.7, respectively) $[t(20)=5.86, p<.01]$. As in Experiment 1 , contextual cuing was still able to occur after the search had begun.

To take a more detailed look at the contextual learning process, we analyzed the distribution of the number of fixations needed to find the target using a four-way ANOVA, with block (8), configuration (repeated or new), fixations until the target was found (1-12), and onset presence as factors. As in Experiment 1, fixations were not evenly distributed $[F(11,209)=19.82, p<.01]$, and the type of configuration affected the distribution of fixations $[F(1,19)=$ 12.77, $p<.01]$. As can be seen in Figure 9, the number of fixations and the type of configuration interacted $[F(11,209)=2.515, p<.01]$, with fewer fixations required to find the target when the subjects viewed repeated configurations. Unlike Experiment 1, block and fixation interacted $[F(77,1463)=1.53, p<.01]$. It can be clearly seen in Figure 10 that fewer fixations were needed to find the target as the subjects became more experienced with the task.

Recognition test. Data from 3 subjects were lost because of a computer error. As in Experiment 1, the subjects were more likely to rate the repeated configurations as familiar than they were to rate the new configurations as familiar $(M \mathrm{~s}=2.78$ and 3.61, respectively, with $1=$ $100 \%$ certain and $5=$ never $)[t(17)=4.28, p<.01]$. As in Experiment 1, no significant correlation was found between the contextual cuing benefits (repeated - new re- sponse times for nononset trials) or the proportional cuing benefits [(repeated - new)/repeated response times for nononset trials] and the degree of recognition.

\section{Discussion}

The results of Experiment 2 suggest that contextual cuing is able to override capture by abrupt onsets. Abrupt onsets slowed response times only when the subjects viewed new configurations and were less effective at increasing the number of fixations when the subjects viewed repeated displays. In addition, the initial fixation was less likely to land on the onset when the subjects viewed a repeated configuration. In contrast, onsets had almost no effect on contextual cuing. Onsets did increase the number of fixations needed to find the target equally for both types of configurations, but onsets did not affect the probability that the first fixation would land on the target and only affected response times when the subjects viewed new configurations. In addition, contextual cuing was still able to occur even when the eyes went initially to the onset.

Taken as a whole, the influence of contextual cuing on attentional capture by onsets suggests that contextual cuing has a higher priority or is otherwise able to lock out attentional capture by onsets. However, it must be kept in mind that contextual cuing had up to a 1 -sec head start over the onsets in Experiment 2. That is, our use of premasks that shared the same arrangement as the eventual stimulus display might have allowed contextual cuing to take hold even before the stimuli and the onset were presented.

One way to explain how contextual cuing can override onsets without giving contextual cuing a special status is to use a horse-race analogy to explain the process by which contextual cuing or abrupt onsets gain control of attention. If the first mechanism to gain control of attention is able to keep other mechanisms from gaining access, then the

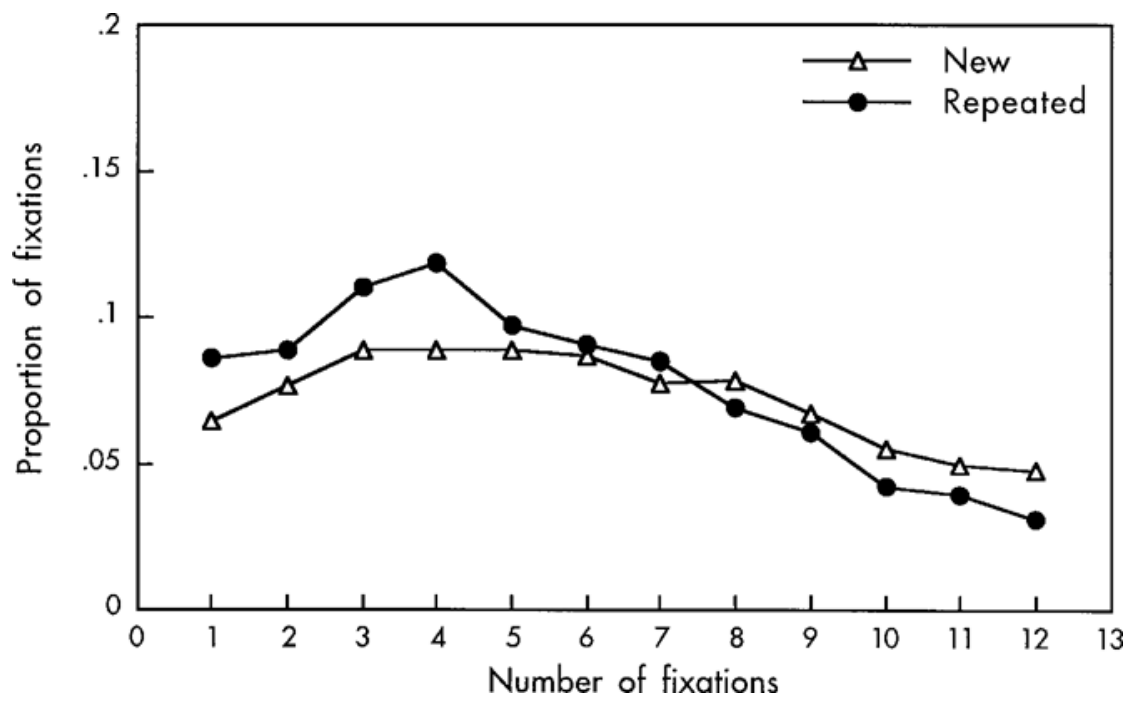

Figure 9. The probability density functions for the number of fixations needed to find the target for the new and repeated displays of Experiment 2. 


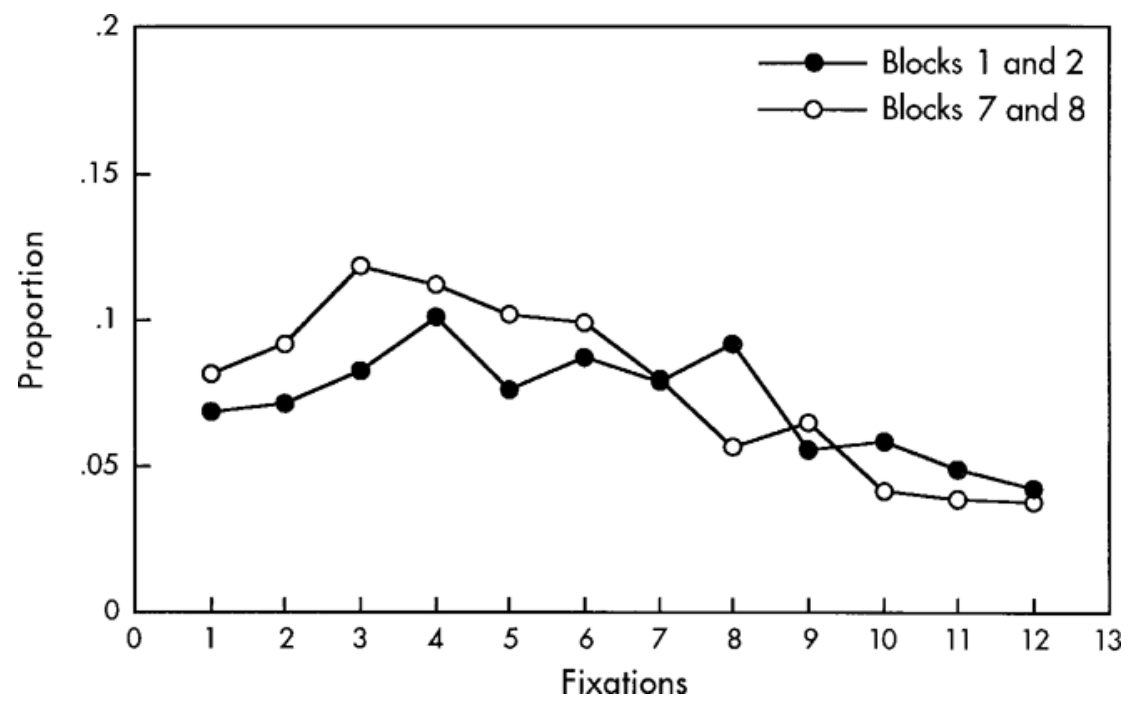

Figure 10. The probability density functions for the number of fixations needed to find the target for Blocks 1 and 2 and Blocks 7 and 8 of Experiment 2. As the subjects gained experience with the task, fewer fixations were needed to find the target.

first mechanism to win the race will win the exclusive rights of attentional control. Given that contextual information was present in our displays before the appearance of the onset, contextual cuing should have been more likely to win the horse race, although the different presentation times of contextual information and abrupt onsets make it difficult to determine the speeds at which contextual cuing and onset capture occur.

The horse-race analogy can also neatly explain why contextual cuing was able to occur even after the eyes initially went to the onset. If the mechanism that responds to onsets sends out a transient signal, whereas contextual cuing sends out a continuous signal (i.e., due to the activation of memory representations of the repeated configurations), then contextual cuing would still be able to guide attention after the onset mechanism has guided the eyes to the onset.

\section{GENERAL DISCUSSION}

Contextual cuing is a phenomenon in which the recognition of global configurations (Chun \& Jiang, 1998) or movements (Chun \& Jiang, 1999) guides attention to a probable location of interest. What has not been clear is how contextual cuing operates. Contextual cuing appears to be an automatic process that does not require explicit recognition of patterns that have occurred previously: The subjects who had reported being aware of repeated patterns showed magnitudes of contextual cuing similar to those reported by the subjects who had not (Experiments 1 and 2; Chun \& Jiang, 1998). This lack of a benefit for explicit recognition would suggest that contextual cuing occurs rapidly before conscious recognition can help in guidance.

Although contextual cuing develops rather quickly, the behavioral data have been less clear about the speed at which contextual cuing occurs. For example, Chun and Jiang (1998) found that search through repeated displays averaged around $27 \mathrm{msec} / \mathrm{item}$ by the end of an experimental session. Clearly, contextual cuing does not always guide attention immediately to the target. This leaves open several possibilities as to the nature of the cuing imprecision. Part of the failure could be due to an imperfect recognition process, such that recognition does not occur on every trial or is completed after search has begun. Another possibility is that the guidance mechanism is imprecise, and, even if recognition is perfect, the guidance mechanism might guide attention in the general direction of the target rather than straight to the target location.

We addressed these questions in Experiment 1 by monitoring the subjects' search patterns through the use of an eye tracker. One important difference between our methodology and that used by Chun and Jiang (1998) is that our displays were preceded by premasks arranged identically to the stimuli that appeared later in the stimulus display. Conceivably, this could have allowed contextual recognition to take place even before the stimuli had appeared. Even with this advantage, contextual recognition was far less than perfect, and the eyes went immediately to the target on only $11.3 \%$ of the trials. This looks less impressive when one takes into account that the target was the first item examined on $7.1 \%$ of the new configuration trials yet was still greater than the chance probability that the eyes initially landed on the target $(8.33 \%)$.

However, recognition is not all or none, and the behavioral cuing benefits do not rest solely on the small numbers of trials in which the target was the first item examined. Repeated displays still showed a benefit over the new displays even when the eyes did not go immediately to the target.

In contrast to the imperfect recognition, guidance was always accurate. ${ }^{3}$ When the eyes did not immediately go 
to the target, the first fixation was no closer to the target when the subjects viewed the repeated displays than when they viewed the new displays. That is, when recognition occurred, the eyes were guided directly to the target; otherwise, the eyes went to another item in the display, with no bias toward the target.

A picture of contextual cuing emerges in which guidance is highly accurate but in which recognition occurs infrequently and sometimes does not occur until later in the search process. To get a better idea of how contextual cuing works, in Experiment 2 we contrasted contextual cuing's memory-based guidance with stimulus-driven attentional capture by abrupt onsets. Contextual cuing was able to override capture by onsets, but this does not necessarily mean that contextual cuing is a special form of guidance with a higher priority than onsets.

Rather, the interaction between contextual cuing (i.e., new and repeated configurations) and abrupt onsets (i.e., presence and absence of abrupt onsets) during the search process can be explained by a horse-race model in which the first mechanism to take control of the guidance mechanism is able to control attention. Contextual cuing could still occur later in the search process, even in instances in which the eyes went initially to the onset; this suggests that, after the onset capture mechanism was finished guiding attention to the onset, contextual cuing was then able to take control.

\section{REFERENCES}

Chun, M. M., \& JiANG, Y. (1998). Contextual cueing: Implicit learning and memory of visual context guides spatial attention. Cognitive Psychology, 36, 28-71.

Chun, M. M., \& JIANG, Y. (1999). Top-down attentional guidance based on implicit learning of visual covariation. Psychological Science, 10, 360-365.

Deubel, H., \& Schneider, W. X. (1996). Saccade target selection and object recognition: Evidence for a common attentional mechanism. Visual Research, 36, 1827-1837.

Folk, C. L., \& Remington, R. [W.] (1998). Selectivity in distraction by irrelevant featural singletons: Evidence for two forms of attentional capture. Journal of Experimental Psychology: Human Perception \& Performance, 24, 847-858.

Folk, C. L., \& Remington, R. [W.] (1999). Can new objects override attentional control settings? Perception \& Psychophysics, 61, 727-739.

Folk, C. L, Remington, R. W., \& Johnston, J. C. (1992). Involuntary covert orienting is contingent on attentional control settings. Journal of Experimental Psychology: Human Perception \& Performance, 18, 1030-1044.

Hoffman, J. E., \& Subramaniam, B. (1995). The role of visual attention in saccadic eye movements. Perception \& Psychophysics, 57, 787-795.

Irwin, D. E., Colcombe, A. M., Kramer, A. F., \& Hahn, S. (2000). Attentional and oculomotor capture by onset, luminance, and color singletons. Vision Research, 40, 1443-1458.
Kramer, A. F., Hahn, S., Irwin, D. E., \& Theeuwes, J. (1999). Attentional capture and aging: Implications for visual search performance and oculomotor control. Psychology \& Aging, 14, 135-154.

Kramer, A. F., Hahn, S., Irwin, D. E., \& Theeuwes, J. (2000). Age differences in the control of looking behavior: Do you know where your eyes have been? Psychological Science, 11, 210-217.

LogAN, G. D. (1988). Towards an instance based theory of automatization. Psychological Review, 95, 492-527.

LogAN, G. D. (1998). What is learned during automatization? II. Obligatory encoding of spatial location. Journal of Experiment Psychology: Human Perception \& Performance, 24, 1720-1736.

Logan, G. D.,TAYlor, S. E., \& ETherton, J. L. (1999). Attention and automaticity: Toward a theoretical integration. Psychological Research, 62, 165-181.

Martin-Emerson, R. \& Kramer, A. F. (1997). Offset transients modulate attentional capture by sudden onsets. Perception \& Psychophysics, 59, 739-751.

Miller, J. (1989). The control of attention by abrupt visual onsets and offsets. Perception \& Psychophysics, 45, 567-571.

Theeuwes, J., Kramer, A. F., Hahn, S., \& Irwin, D. E. (1998). Our eyes do not always go where we want them to go: Capture of the eyes by new objects. Psychological Science, 9, 379-385.

Theeuwes, J., Kramer, A. F., Hahn, S., Irwin, D. E., \& Zelinsky, G. J. (1999). Influence of attentional capture on eye movement control. Journal of Experimental Psychology: Human Perception \& Performance, 25, 1595-1608.

Wolfe, J. M., Cave, K. R., \& Franzel, S. L. (1989). Guided search: An alternative to the feature integration model for visual search. Journal of Experimental Psychology: Human Perception \& Performance, 15, 419-433.

Yantis, S., \& Egeth, H. (1999). On the distinction between visual salience and stimulus-driven attentional capture. Journal of Experiment Psychology: Human Perception \& Performance, 25, 661-676.

YANTIS, S., \& JoNidES, J. (1984). Abrupt visual onsets and selective attention: Evidence from visual search. Journal of Experiment Psychology: Human Perception \& Performance, 10, 601-621.

YAnTIS, S., \& Jonides, J. (1990). Abrupt visual onsets and selective attention: Voluntary versus automatic allocation. Journal of Experimental Psychology: Human Perception \& Performance, 16, 121-134.

\section{NOTES}

1. Although some of Chun and Jiang's (1998) subjects reported being aware that some of the displays had been repeated, no difference was found between their performance and the performance of subjects that were unaware of the repetitions.

2. Although there are global patterns that are common to the repeated configurations, in an experiment with 12 repeated and 12 unique configurations, an instance of repeated global patterns occurs on only $0.042 \%$ of the trials.

3. It could be argued that recognition was always accurate and it was guidance that was imperfect; however, if this was the case, then a bias should have been found in the distance of the first fixation from the target when the eyes did not first land on the target. In addition, the fact that contextual cuing benefits can still be found when the eyes did not immediately go to the target suggests that it is recognition, rather than guidance, that is imperfect.

(Manuscript received March 1, 2000; revision accepted for publication January 23, 2001.) 